\title{
Population III Wolf-Rayet Stars in the CAK Regime
}

\author{
A. J. Onifer \\ Los Alamos National Laboratory, PO Box 1663, MS T087, Los Alamos, NM 87544
}

\begin{abstract}
Wolf-Rayet (WR) stars near solar metallicity are believed to be driven by radiation pressure on the UV spectral lines of metal ions. As the metallicity decreases so does the line opacity, therefore the mass-loss rate. However, since the composition of a WR atmosphere is determined by the burn products of the core, there is a lower limit on the line opacity and therefore the mass-loss rate - of a WR star, even in a star with zero initial metallicity. This presentation is the result of attempt to calculate the mass-loss rate of a Population III-type WO star using a modified version of the CAK approximation. I find that $n_{e} \geq 10^{13} \mathrm{~cm}^{-3}$ and $0.5 \leq \Gamma \leq 0.7$ give the most plausible results, with the resulting mass-loss rate between $2 \times 10^{-9}$ $\mathrm{M}_{\odot} \mathrm{yr}^{-1}$ and $3 \times 10^{-8} \mathrm{M}_{\odot} \mathrm{yr}^{-1}$.
\end{abstract}

Keywords: Wolf-Rayet stars, Stellar winds, Mass loss, Radiative transfer, Population III stars

\section{INTRODUCTION}

At low metallicity line-driven Wolf-Rayet (WR) winds exhibit smaller mass-loss rates than WR stars near solar metallicity. Since WR winds are significant contributers of chemical enrichment and energy deposition at all metallicities, understanding the physics of wind driving at low metallicity is important.

WR winds have been studied computationally via radiative transfer [1] or radiation hydrodynamics simulations [2]. As a complement to these analyses, this study uses simple analytic models based on a modified version of CAK theory [3] to determine the effects of a Population III abundance profile on WR wind driving. To keep the results as general as possible, model-specific parameters such as the velocity law and the location of the critical point are not specified.

Recently Vink and de Koter [4] explored the metallicity dependence of WR mass loss using a Monte Carlo approach with CAK-type line driving for stars at metallicity down to $Z / Z_{\odot}=10^{-5}$. They found that the massloss rate flattens at low metallicity $\left(Z / Z_{\odot}<10^{-3}\right)$ because the self-enrichment of $\mathrm{N}$ and $\mathrm{He}$ in WN stars and $\mathrm{C}$ in WC stars provide the lines needed for wind driving. They found $\dot{M} \approx 10^{-8} \mathrm{M}_{\odot} y r^{-1}$ for low metallicity WN stars and $\dot{M}=1.4 \times 10^{-7} \mathrm{M}_{\odot} \mathrm{yr}^{-1}$ for low metallicity WC stars. With these results in mind, this presentation provides results for a WO star at the extreme of zero background metallicity, varying the luminosity (via the Eddingtom parameter $\Gamma$ ) and the electron density.

\section{CAK THEORY AND MODIFICATIONS}

The first successful analytic models of line-driven winds were developed by Castor et al. [3] (hereafter CAK) to model the winds of OB stars. CAK theory models wind driving via radiation pressure on a large number of UV lines. It relies on the Sobolev approximation [5], which assumes a rapidly accelerating wind such that a photon's Sobolev length $L_{s o b}=v_{t h}(d v / d r)^{-1}$, where $v_{t h}$ is the thermal speed and $d v / d r$ is the gradient of the wind velocity, is much smaller than the mean free path between lines.

In the CAK model the radiation pressure on the lines is expressed as a ratio of the radiative acceleration of the lines $g_{L}$ to the radiative acceleration of the free electrons $g_{e}$ using the so-called force multiplier $M(t)$,

$$
M(t)=\frac{g_{L}}{g_{e}} .
$$

The force multiplier is then parameterized by the relation

$$
M(t)=k t^{-\alpha},
$$

where $k$ and $\alpha$ are determined from the line strength distribution, and $\alpha$ in particular is a number between 0 and 1 that is related to the fraction of optically thick lines, thus $\alpha=1$ means all lines are optically thick over $L_{s o b}$.

The details of the model used in this analysis, which include the effects of frequency redistribution due to line branching and bound-free opacity, can be found in Onifer and Gayley [6] (hereafter OG) and references therein. The following are some highlights.

In CAK theory the momentum equation at the critical point in units of the effective gravity can be written (see OG, Sec. 2 and Eq. 27),

$$
1+y_{c}=\frac{\Gamma}{1-\Gamma} M(t) F_{N I D} F_{r e d},
$$

where the first term on the left-hand side represents the effective gravity, $\Gamma$ is the Eddington parameter, $F_{N I D}$ is a correction for multiple scattering [7], $F_{\text {red }}$ is a correction for frequency redistribution due to line branching and 
bound-free opacity, and $y$ is the inertia scaled to the effective gravity,

$$
y_{c}=\frac{r_{c}^{2} v_{c}(d v / d r)_{r_{c}}}{G M_{*}(1-\Gamma)},
$$

where $r_{c}$ and $v_{c}$ are the radius and velocity at the critical point and $M_{*}$ is the stellar mass.

\section{MODEL SETUP}

One cannot hope to model analytically all the complexity that a radiative tranfer or radiation hydrodynamics code is capable of modeling, so some simplifying assumptions must be made. The wind is assumed to be spherically symmetric, smooth on scales larger than $L_{s o b}$, and time steady, and the star is assumed to be nonrotating.

At low $Z$ a WR star is more likely to evolve to the WO phase than at high $Z$ because the small amount of mass lost in the WN phase allows more production of $\mathrm{O}$ via $\alpha$-particle capture of $\mathrm{C}$ before the overlying envelope is stripped. The WO phase can be defined by the relation $\left(N_{C}+N_{O}\right) / N_{H e}>1.0$, where $N_{i}$ is surface number fraction [8]. The surface abundances are based on the SMC WO star Sand 1 studied by Kingsburgh et al. [9]. The mass fractions of the included elements are $Y=0.21$, $X_{C}=0.51, X_{O}=0.25, X_{N e}=0.02$. The Ne is entirely ${ }^{22} \mathrm{Ne}$, which is a product of the He-burning process. All ion states of each element are included. The Sobolev opacities are calculated using oscillator strengths from the Opacity Project [10]. Details of the opacity calculation can be found in OG.

The mass of the star is $5 M_{\odot}$, also based on Sand 1 . This is may be a bit small even for a highly-evolved Population III WR star due to the lower mass-loss rates of such stars, so the mass-loss rates should be thought of as lower limits. The terminal speed $v_{\text {inf }}$ is set such that $v_{\text {inf }}-v_{c}=4700 \mathrm{~km} \mathrm{~s}^{-1}$. Kingsburgh et al. [9] report $v_{\text {inf }}=4200 \mathrm{~km} \mathrm{~s}^{-1}$, which gives $v_{c}=500 \mathrm{~km} \mathrm{~s}^{-1}$. This reflects the large terminal speeds of WO stars. $\dot{M}$, though ultimately has a small dependence on $v_{\text {inf }}$, since $\dot{M}$ is set by conditions near the surface at the critical point and $v_{\text {inf }}$ is set by the global wind conditions. The temperature at the critical point $T_{c}=1.3 \times 10^{5} \mathrm{~K}$. Two sets of models are run. One set assumes complete frequency redistribution (CRD). The other relaxes the CRD assumption by accounting for for changes in the wind ionization structure due to the changing wind temperature over a thermalization length, or length beyond which frequency redistribution affects the spectral flux profile [11]. In this model the thermalization length is such that the final temperature is $T=4.0 \times 10^{4} \mathrm{~K}$. The electron density $n_{e}$ is set to either $10^{12} \mathrm{~cm}^{-3}, 10^{13} \mathrm{~cm}^{-3}$ or $10^{14} \mathrm{~cm}^{-3}$. Setting a constant $n_{e}$ implies that the velocity curve and/or the

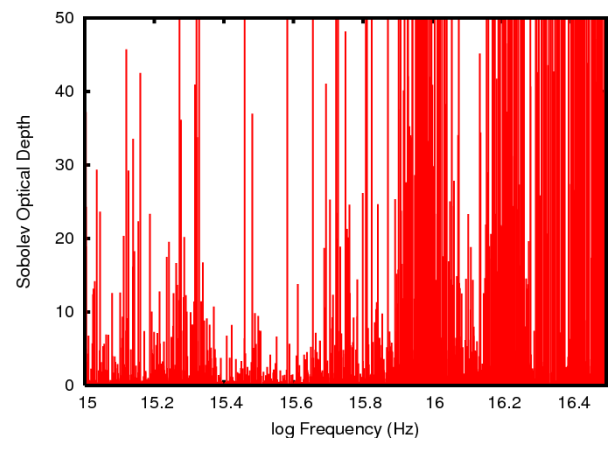

FIGURE 1. Sobolev optical depth as a function of frequency at solar metallicity.

stellar radius is different for each model, though neither as specified explicitly, as this study is concerned more with modeling a representative star, rather than a grid of specific stars.

\section{RESULTS}

Not surprisingly, the lack of metals, especially of iron group ions, causes a large drop in the line opacity. Figure 1 shows the Sobolev optical depth spectrum for a solar metallicity star with stellar parameters similar to those explored in this paper. Figure 2 shows the Sobolev optical depths for the $Z=0$ case in CRD. Clearly there is much less opacity to drive the wind.

The mass loss rates and wind parameters are shown in table 1. Only models that produced physical results (e.g., $\left.F_{\text {red }}<1\right)$ are shown. None of the CRD models were able to produce a wind. Thus for the given parameter ranges a CAK-type wind requires a finite thermalization length. As the wind density drops, $M(t)$ rises, meaning when the continuum is weaker, the lines take on a larger fraction of the momentum driving. The models for which $10<$ $M(t)<50$ are most similar to Population I WR stars, and are probably the most plausible for Population III WR stars. Of these, the mass-loss rate is in the range $2 \times 10^{-9}$ $\mathrm{M}_{\odot} \mathrm{yr}^{-1}<\dot{M}<3 \times 10^{-8} \mathrm{M}_{\odot} \mathrm{yr}^{-1}$. Compared to Vink and de Koter's low-Z mass-loss rate $\dot{M} \approx 10^{-7} \mathrm{M}_{\odot} \mathrm{yr}^{1}$, my rates are small, though the results are not directly comparable due to the difference in stellar mass and the WO-like abundances of models in this presentation.

\section{CONCLUSIONS}

A small parameter study of an analytic, modified CAKtype model of Population III WO-type stellar winds has been performed. The most plausible results were found for $\Gamma=0.5$ and $\Gamma=0.7$ and $10^{13} \mathrm{~cm}^{-3}<n_{e}<10^{14}$ 
TABLE 1. Results for model calculations

\begin{tabular}{ccrccl}
\hline $\mathbf{n}_{\mathbf{e}}\left(\mathbf{c m}^{-\mathbf{3}}\right)$ & $\Gamma$ & $\tau_{\mathbf{e}}$ & $\mathbf{t}$ & $\mathbf{M}(\mathbf{t})$ & $\dot{\mathbf{M}}\left(\mathbf{M}_{\odot} \mathbf{y r}^{-\mathbf{1}}\right)$ \\
\hline $1 \times 10^{14}$ & 0.3 & 5 & $3.6 \times 10^{-5}$ & 96.3 & $7.3 \times 10^{-9}$ \\
$1 \times 10^{14}$ & 0.3 & 10 & $4.1 \times 10^{-5}$ & 91.5 & $8.6 \times 10^{-9}$ \\
$1 \times 10^{14}$ & 0.5 & 5 & $1.0 \times 10^{-4}$ & 6.7 & $1.6 \times 10^{-8}$ \\
$1 \times 10^{14}$ & 0.5 & 10 & $1.1 \times 10^{-4}$ & 12.0 & $1.9 \times 10^{-8}$ \\
$1 \times 10^{14}$ & 0.7 & 2 & $5.0 \times 10^{-4}$ & 15.0 & $1.9 \times 10^{-8}$ \\
$1 \times 10^{14}$ & 0.7 & 5 & $7.1 \times 10^{-4}$ & 13.5 & $2.5 \times 10^{-8}$ \\
$1 \times 10^{14}$ & 0.7 & 10 & $8.9 \times 10^{-4}$ & 10.6 & $2.8 \times 10^{-8}$ \\
\hline $1 \times 10^{13}$ & 0.3 & 5 & $1.2 \times 10^{-5}$ & 134 & $8.6 \times 10^{-10}$ \\
$1 \times 10^{13}$ & 0.3 & 10 & $1.7 \times 10^{-5}$ & 98.9 & $1.0 \times 10^{-9}$ \\
$1 \times 10^{13}$ & 0.5 & 2 & $5.8 \times 10^{-5}$ & 49.7 & $2.2 \times 10^{-9}$ \\
$1 \times 10^{13}$ & 0.5 & 5 & $7.5 \times 10^{-5}$ & 31.5 & $3.5 \times 10^{-9}$ \\
$1 \times 10^{13}$ & 0.5 & 10 & $8.6 \times 10^{-5}$ & 26.4 & $4.5 \times 10^{-9}$ \\
$1 \times 10^{13}$ & 0.7 & 2 & $1.5 \times 10^{-4}$ & 17.2 & $6.5 \times 10^{-9}$ \\
$1 \times 10^{13}$ & 0.7 & 5 & $1.7 \times 10^{-4}$ & 15.8 & $9.5 \times 10^{-9}$ \\
$1 \times 10^{13}$ & 0.7 & 10 & $1.8 \times 10^{-4}$ & 15.1 & $1.2 \times 10^{-8}$ \\
\hline $1 \times 10^{12}$ & 0.3 & 2 & $2.8 \times 10^{-6}$ & 377 & $2.8 \times 10^{-10}$ \\
$1 \times 10^{12}$ & 0.3 & 5 & $3.1 \times 10^{-6}$ & 394 & $3.9 \times 10^{-10}$ \\
$1 \times 10^{12}$ & 0.3 & 10 & $3.3 \times 10^{-6}$ & 326 & $4.6 \times 10^{-10}$ \\
$1 \times 10^{12}$ & 0.5 & 2 & $5.1 \times 10^{-6}$ & 222 & $7.2 \times 10^{-10}$ \\
$1 \times 10^{12}$ & 0.5 & 10 & $5.8 \times 10^{-6}$ & 225 & $1.1 \times 10^{-9}$ \\
\hline & & & & &
\end{tabular}

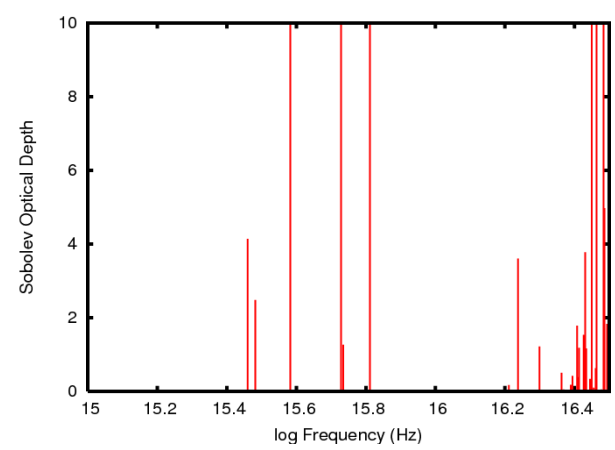

FIGURE 2. The same as figure 1 but at $\mathrm{Z}=0$ and CRD.

$\mathrm{cm}^{-3}$, with mass-loss rates in the range $2 \times 10^{-9} \mathrm{M}_{\odot}$ $\mathrm{yr}^{-1}<\dot{M}<3 \times 10^{-8} \mathrm{M}_{\odot} \mathrm{yr}^{-1}$.

A similar analysis could be done for WN stars, a phase through which all WR stars pass. WN stars will have an even lower mass-loss rate, as the only line driving would be provided by $\mathrm{He}$ and a relatively small amount of $\mathrm{N}$. These results will be used in a study of mass-loss as a function of small but nonzero metallicity.

\section{ACKNOWLEDGMENTS}

The author would like to thank Alexander Heger for helpful discussions. This work was performed under the auspices of the U. S. Department of Energy by the Los
Alamos National Security (LANS), LLC under contract No. DE-AC52-06NA25396.

\section{REFERENCES}

1. D. J. Hillier, and D. L. Miller, ApJ 496, 407-+ (1998).

2. G. Gräfener, and W.-R. Hamann, $A \& A$ 432, 633-645 (2005).

3. J. I. Castor, D. C. Abbott, and R. I. Klein, ApJ 195, 157-174 (1975).

4. J. S. Vink, and A. de Koter, A\&A 442, 587-596 (2005).

5. V. V. Sobolev, Moving envelopes of stars, Cambridge: Harvard University Press, 1960.

6. A. J. Onifer, and K. G. Gayley, ApJ 636, 1054-1066 (2006).

7. K. G. Gayley, S. P. Owocki, and S. R. Cranmer, ApJ 442 , 296-310 (1995).

8. M. J. Barlow, and D. G. Hummer, "The WO Wolf-Rayet stars," in Wolf-Rayet Stars: Observations, Physics,

Evolution, edited by C. W. H. de Loore, and A. J. Willis, 1982, vol. 99 of IAU Symposium, pp. 387-392.

9. R. L. Kingsburgh, M. J. Barlow, and P. J. Storey, $A \& A$ 295, 75-100 (1995).

10. N. R. Badnell, M. A. Bautista, K. Butler, F. Delahaye, C. Mendoza, P. Palmeri, C. J. Zeippen, and M. J. Seaton, MNRAS 360, 458-464 (2005).

11. L. B. Lucy, and D. C. Abbott, ApJ 405, 738-746 (1993). 